\title{
A Review Paper on Facial Detection Technique Using Pixel and Color Segmentation
}

\author{
Chandrshekar.S.Patil \\ Assistant Professor \& TC Department \\ S.G.D.C.O.E, Jalgaon
}

\author{
A.J.Patil, PhD. \\ Principal \\ S.G.D.C.O.E., Jalgaon
}

\begin{abstract}
In this paper a review is being carried out in the field of facial recognition and automatic human face detection. It has become a very popular tool in wide spaces of applications including the criminal investigation department in the forensic sciences along with the presentation of a biometric tool for the users to provide their unique identity. The databases for the face detection are a more genuine and easily accessible tool in carrying out the identification aspect when we don't have enough evidences for the finger prints collection. A review has been made in this field while considering the pixel operation and color segmentation based approach.
\end{abstract}

Keywords- Face Detection, Color Space, Support Vector Machine, Facial Feature Area, Criminal Justice Application, Gender Classifications.

\section{HISTORY}

Researches in automatic face recognition started in the 1960s. There has been significant progress in the recent years that includes a number of face recognition and modeling systems.

Face recognition system is valuable for the human identification when the finger print identification goes impracticable.

The history of the face recognition dates back to the advent of photography. Both the Government and private organizations have kept collection of photos of people because it made their way onto personal identification from passports to the identity cards issued by the Government or other agencies.

The first attempt to automated facial recognition approach consisted of checking the measurements between different facial features such as the corners of the eyes, the hair lines etc. This attempt was not that much successful. Towards the end of 1980s, the eigenfaces 2 techniques prompted more intense researches which were used to find a face in a photo and to compare the images of field has reached up to that point where the operational use of facial recognition on high resolution frontal image was now feasible.

\section{AUTOMATIC HUMAN FACE DETECTION (AHFD)}

It is a digital technology which determines the presence of the human faces in arbitrary colored digital images. AHFL has the task to find the location and sizes of a known number of the faces which is based on newer algorithms to solve the more general and difficult problem of multi view of face detection. Gender classification finds out whether the given facial image belongs to the male or female. The detection principle is mainly based on the selection of the color space to detect the skin tone face detection and gender classification methods.

Face detection is a feature based approach in which face geometry is taken which includes face shape and other facial features like mouth, eyes, nose etc. The algorithm requires 2$\mathrm{D}$ images whose threshold values of intensities are taken into consideration in the measurement of the number of the pixels to get the entire face feature area.

\section{METHODOLOGY}

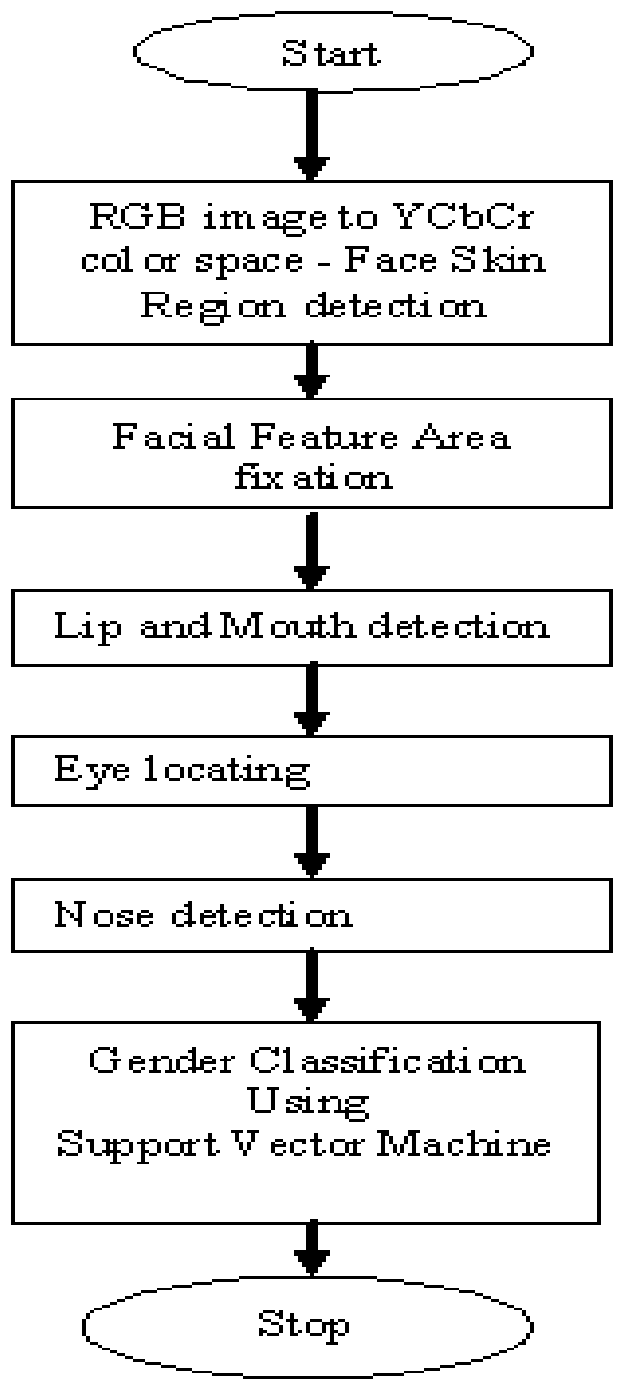

Fig 1: Flow chart 


\subsection{Face detection}

The $\mathrm{YCbCr}$ color space is used to detect the skin region on the given input face image. Processing color is faster than any other feature. So, skin color detection is firstly performed on the input color image to reduce the computational complexity. There are non linear relation between chrominance $(\mathrm{CbCr})$ and luminance( $\mathrm{Y})$ of skin color in various luminance regions (brightness component).

In the color detection processs, each pixel of the image is classified either as skin or non skin based on its color components [2].

The gamma corrected RGB value is determined as

Gamma $\mathrm{RGB}=(\mathrm{c} 1 *$ input RGB image $){ }^{\mathrm{c} 2}+\mathrm{c} 3$

Where $\mathrm{c} 1=1.0, \mathrm{c} 2=1.0, \mathrm{c} 3=0.0$

$\mathrm{Y}=(0.299 *($ gamma RGB $[0, \mathrm{i}, \mathrm{j}]-\mathrm{C}))+\mathrm{C}+(0.114 *($ gamma RGB $[2, \mathrm{i}, \mathrm{j}]-\mathrm{C}))$

$\mathrm{Cb}=(0.564 *($ gammaRGB $[2, \mathrm{i}, \mathrm{j}]-\mathrm{Y}))$

$\mathrm{Cr}=(0.713 *($ gammaRGB $[0, \mathrm{i}, \mathrm{j}]-\mathrm{Y}))$

Where Gamma RGB is the array, 0 represents the red layer and 2 represent the blue layer. The range of $\mathrm{Cb}$ is between -50 and 2 while the range of $\mathrm{Cr}$ is between $10 \& 100$. The given $\mathrm{YCbCr}$ image is converted to one layer gray scale image. This system takes the green layer. The face boundary estimation is carried out by the center part of the gray image face photo. The skin image is estimated based on the threshold gray level between $20 \& 80$. Similarly, the outer boundary is also determined [2].

\subsection{Facial feature detection}

This technique involves the facial feature area estimation, lip $\&$ mouth determination, right and left eye locating and noise estimation process. The middle part of the image having the constant value as (width/2 or height/2) or $\left(\mathrm{X}_{\text {mid }}, \mathrm{Y}_{\text {mid }}\right)$ is formed. Than all the pixels that lie between the region of

$\left(\mathrm{Y}_{\text {mid }}-(\right.$ height of image/5) -10$)$ and

$\left(\mathrm{Y}_{\text {mid }}+\right.$ height of image/5)

And

$\left(\mathrm{X}_{\text {mid }}-\left(\right.\right.$ width of image/5) and $\left(\mathrm{X}_{\text {mid }}+(\right.$ width of image/5)

(6)

constitutes the facial feature area enclosing the eyes, nose, mouth. The lip is estimated by searching a point at the left bottom and then slowly moving upwards. The scan is performed column wise and wherever a pixel is found, the search stops. Height of the mouth area is from (detected lip point -15 row pixels) to (detected lip point +15 row pixels) and width of the mouth area from (detected lip point +45 column pixels). Similarly, a point is found that might be the left most pixel of the right eye and from that detected point, the width and height of the right eye is estimated. Similarly left eye portion is found.

The width of the nose area is calculated as the difference between the right eye's end point and the left eye's starting point. The height of the nose is estimated by the region that starts at the right eye's endpoint and ends at the detected lip point plus 25 row pixels [2].

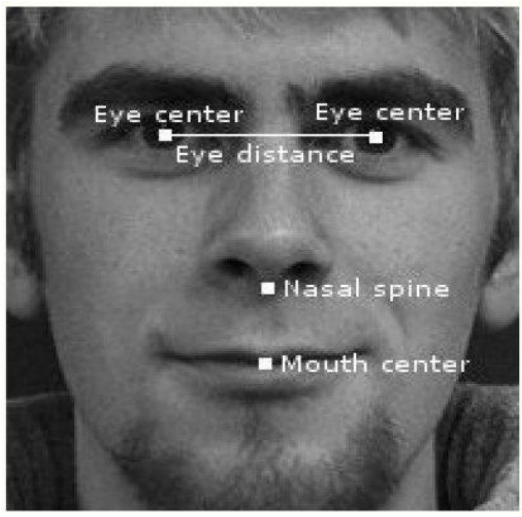

Fig2: Facial Feature Recognition

\subsection{Gender classification}

Support vector machines are based on the concept of the decision plane that defines the decision boundaries. A decision plane separates between a set of objects having different class memberships. The proposed algorithm uses the linear SVM. For all the image pixel values

SVM $[$ index $]=$ Sum of intensity value of 3 layers(RGB) of the pixel in the image

processed $\times 255 \times 3$

-No. of pixels so far

(7)

Where 255 is the maximum intensity value of the gray level intensity and 3 is the total no. of layers. The value in the array SVM[index] is given as the input to the function LEGENDRE( ) that returns the value of the associated Legendre polynomial $\operatorname{Pm}(\mathrm{x})$ where $\mathrm{x}$ is the value evaluated in this expression. Its range should be between $-1 \&+1$. $\mathrm{L}$ is the scaled integer array, $\mathrm{L}>0$ specifies the order of the function. If it specifies, then it is the linear SVM classification If the resultant SVM is greater than the estimated threshold value 0.07 , then the face in the given input image is of male otherwise it is of female.

\section{COLOR BASED SEGMENTATION}

Different ethnic groups have different levels of the melanin \& pigmentation and the range of colors that human facial skin takes on is a subspace of total color space. For this purpose, 3 color spaces have been taken namely HSV, YCrCb and RGB.

1) HSV color space: It is much more intuitive and provides color information in a manner and how human think of colors and how artists typically mix colors. Hue describes the basic purity of the color image whereas saturation gives manner by which pure color is diluted by the white light and value provides the intensity of the color. The most noticeable trend was to was used to derive the following rule used in face skin detection block [3]

$19<\mathrm{H}<240$

Implies that it is not skin otherwise it is skin.

2) For $\mathrm{YCbCr}, 102<\mathrm{Cb}<128$ 
Implies that it is skin, otherwise it is not skin.

3) RGB color space

It is most commonly used basis for color descriptions. It has the negative aspect that each of the coordinates is subjected to luminance effect from the lighting intensity of the environment, it does not provide necessary information whether particular image is skin or not. While experimenting with various thresholds in the RGB space, the following rule worked well in reviewing some necessary pixels.

$0.836 \mathrm{G}-14<\mathrm{B}<0.836 \mathrm{G}+44$

and

$0.79 \mathrm{G}-67<\mathrm{B}<0.78 \mathrm{G}+42$

Implies the skin texture [3].

\section{LOWER PLANE MASKING}

When we remove the lower portion of the image from the consideration to remove the possibility of false texture originating from this region, we are remained with the useful

remaining pixel for the facial detection.

\subsection{Morphological processing}

Applying the open operation after removing the vast majority of pixels, we see little specs throughout the masked image which needs to be removed to speed the future processing which is carried out using a $3 \times 3$ window of all 1 second that will result in the reduction of noisy specs.

\subsection{Removal of blobs and gray scale transformation}

Blobs are the connected group of pixels that remain at this stage as the subjects were standing nearby closer to each other. Hence their head sizes (measured by the number of pixels) are relatively similar. The largest blobs should be these heads and considerably smaller than larger blobs may be safely assumed to be noisier. By removing these blobs below a threshold size, we can remove even more additional noise. Pixel size of 200 has a good threshold value.

Blob size $<200$ implies the non face blob

Thus we are left with the stage when we don't have any performance degradation but with the additional benefit of the faster system. It will provide with a final preprocessed image [3].

\section{ALGORITHM FOR THE TEMPLATE DESIGN FOR FACE DETECTION}

1. Image is resized through appropriate filtering and sub sampling and the region is selected to be $30 \times 30$ pixels.

2. Convolve the masked gray scale image with the template and normalize the output by the energy in the template.

3. Look for the peaks in the resulting output and compare them to a given range of thresholds.

4. Pixels that fall within the threshold range are marked as such to prevent the occurrence of false detection.
5. The threshold range is reduced to present lower limit and then another stage of convolving is applied. If the lower limit is reached then proceed to the next level.

6. To detect the larger scale faces, template is enlarged and thresholds are reset to the upper limit and again the whole process is carried out.

7. Finally when the upper stage is reached, quit the process.

We will find that there are the peaks at the location of faces and these peaks are closely related to each other [3].

\section{RESULTS}

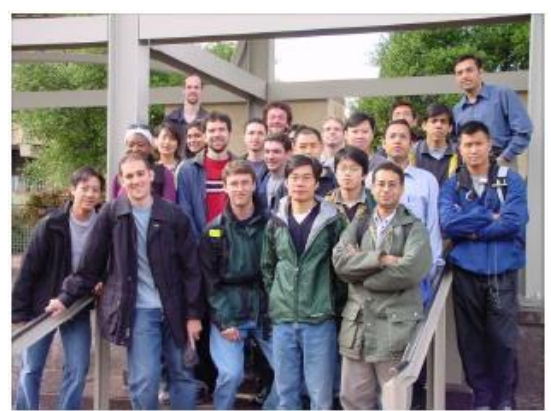

Fig 3:Example Input Training/Testing Image

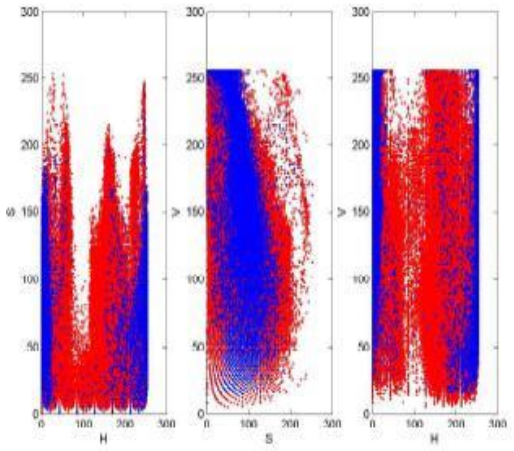

Fig 4:H vs. S vs. plots for face (blue) and non-face (red) pixels

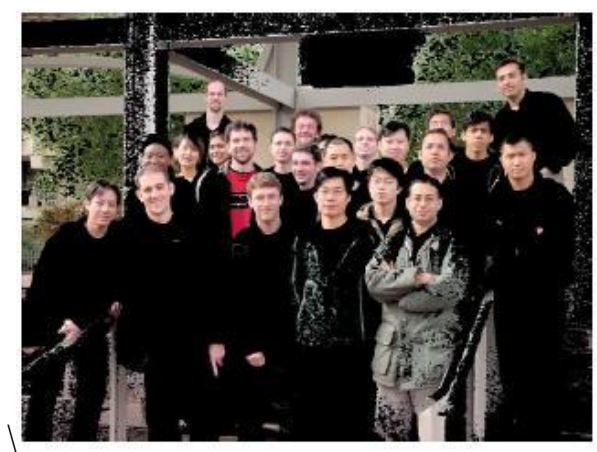

Fig 5:Remaining pixels after applying the HSV segmentation rule 


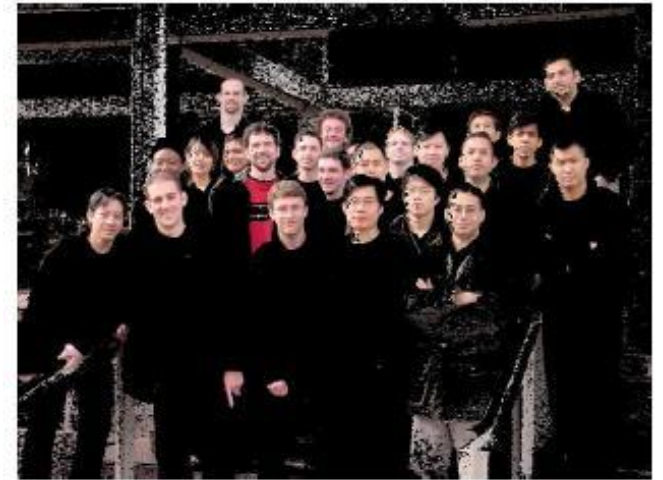

Fig 6:Remaining pixels after applying the $\mathrm{YCbCr}$ segmentation rule

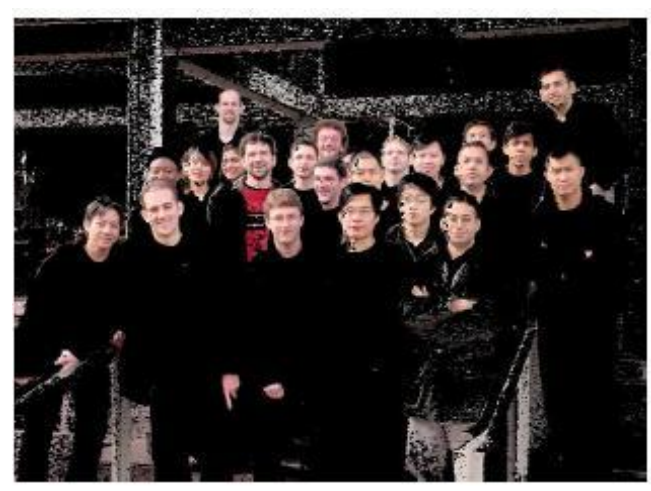

Fig 7:Remaining pixels after applying the first RGB segmentation rule

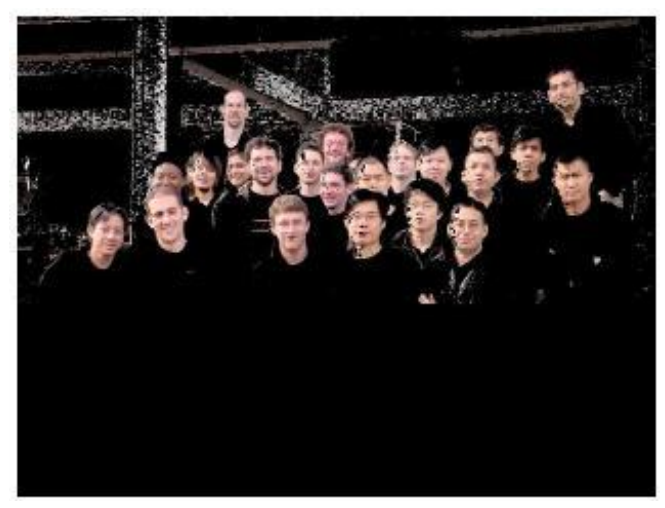

Fig 8:Remaining pixels after masking the lower image field

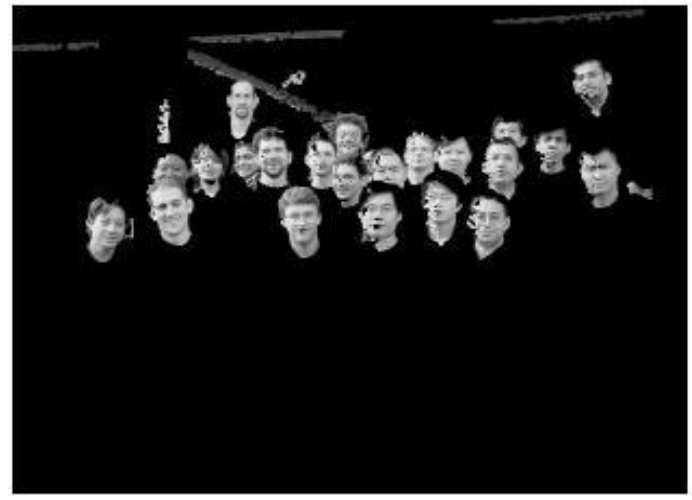

Fig 9:Final pre-processed image after small blob removal and grayscale Transformation

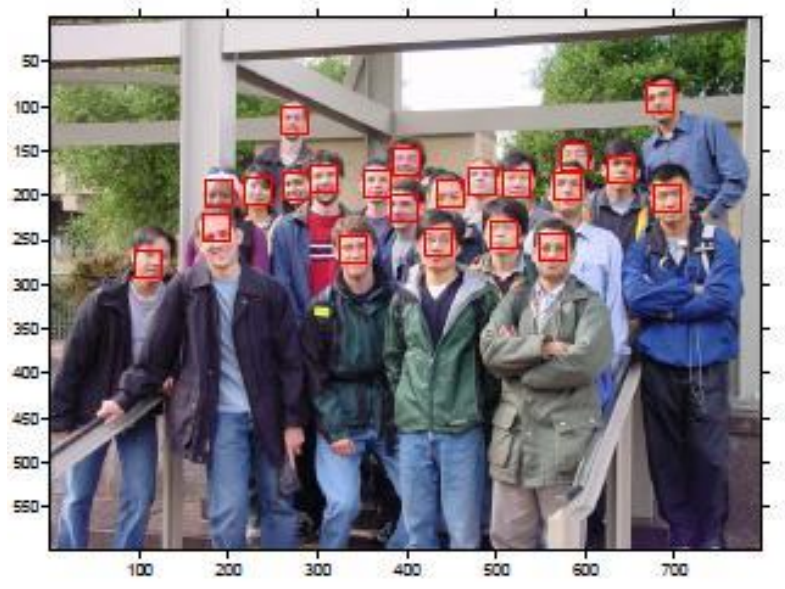

Fig 10:Final result of face detection algorithm

\section{APPLICATIONS}

1. Mostly in the criminal justice application facial recognition provides an alternative method to make sure that the databases do not contain multiple records for a single individual. Thus, it allows people to be identified when it is not possible to take the fingerprints for physical or legal reasons.

2. IDs checks can be carried out on just the faces or the fingerprints; the combination of the two biometric techniques increases the accuracy of the searches and allows reliable decisions to be sent to the required field. Even, if the quality of the external facial images is highly variable, it is possible to compare them with the photograph of the person known to the Police.

\section{REFERENCES}

[1] Eric Hess ''Facial recognition: A valuable tool for Law Enforcement', Forensic magazine, Vol.7/ No.5, November 2010

[2] S.Ravi, S.Wilson, "Face detection with facial features and gender classification based on support vector machine", 2010 IEEE International Conference on Computational Intelligence and computing Research, ISBN: 978818371 3627.

[3] Michael Padilla and Zihong Fan, “EE368 Digital Image Processing Project- Automatic Face Detection using Color Based Segmentation and Template/Energy Thresholding", Department of Electrical engineering, EE368, Stanford University. 